\title{
Pre- and post-therapy nodal status equally affects survival of patients with oesophageal squamous cell carcinoma receiving preoperative chemoradiation
}

\author{
HIROSHI MIYATA, MAKOTO YAMASAKI, SHUJI TAKIGUCHI, KIYOKAZU NAKAJIMA, \\ YOSHIYUKI FUJIWARA, MASAKI MORI and YUICHIRO DOKI
}

\begin{abstract}
Department of Gastroenterological Surgery, Graduate School of Medicine, Osaka University, Osaka 565-0871, Japan
\end{abstract}
Received October 19, 2009; Accepted January 5, 2010

DOI: 10.3892/or_00000768

\begin{abstract}
Patients with deeply invading (T3-T4) oesophageal cancers usually receive chemoradiotherapy with or without surgery. However, the prognostic significance of pre-therapy and post-therapy lymph node (LN) status remains unclear. We studied 195 patients who received chemoradiotherapy for deeply invading oesophageal cancers (T3-4, N0-1, M0). Of these, 105 patients underwent surgery while 90 were treated by chemoradiotherapy alone. Of the 105 surgically treated patients, overall survival was significantly better in $\mathrm{cN} 0$ patients than in $\mathrm{cN} 1$ (3-year survival rate, 65.3 vs. $25.8 \%$, $\mathrm{P}=0.0014)$. This difference was similarly observed in 90 patients who received chemoradiotherapy alone. Patient survival differed significantly among patients with no positive $\mathrm{LN}, 1$ positive $\mathrm{LN}$ and 2-4 positive $\mathrm{LN}$ (3-year survival rate, 57.1 vs. 40.5 vs. $17.6 \%, \mathrm{P}<0.0001)$. However, there was no significant difference in survival between patients with 2-4 positive $\mathrm{LN}$ and $\geq 5$ positive LN. Multivariate analysis identified pre-therapy LN status and the number of involved LNs as the most important independent prognostic factors prior to histopathological tumour regression. In conclusion, pretherapy LN status and the number of post-therapy involved LNs equally affect survival of patients who receive neoadjuvant chemoradiotherapy. Control of systemic metastasis is required, based on pre- and post-therapy LN status.
\end{abstract}

\section{Introduction}

Oesophageal cancer is one of the most intractable gastrointestinal cancers. Locoregional and systemic recurrences remain common even after surgical curative resection, and the 5 -year survival rate ranges from 15 to $39 \%$ (1-4). To improve the prognosis for these patients, multimodal therapy com-

Correspondence to: Dr Hiroshi Miyata, Department of Gastroenterological Surgery, Graduate School of Medicine, Osaka University, 2-2 Yamadaoka, Suita, Osaka 565-0871, Japan

E-mail: hmiyata@gesurg.med.osaka-u.ac.jp

Key words: oesophageal cancer, chemoradiotherapy, lymph node status prising preoperative or postoperative chemotherapy and/or radiotherapy has been developed. Neoadjuvant chemoradiotherapy followed by surgery is widely accepted as one of the most promising strategies for locally advanced oesophageal cancers. The main aim of this strategy is local tumour control, to facilitate complete resection of the primary tumour and decrease locoregional recurrence. Indeed, in several trials, neoadjuvant chemoradiotherapy prior to surgery increased complete resection rates and improved prognosis, compared with surgery alone (5-7). Moreover, two metaanalyses of randomized controlled trials revealed that neoadjuvant chemoradiotherapy followed by surgery improved patient prognosis and reduced locoregional recurrence, compared with surgery alone $(8,9)$.

It is commonly accepted that patients who achieve a pathological complete response after neoadjuvant chemoradiotherapy for locally advanced oesophageal cancers have an improved prognosis (10-13). Moreover, several studies found the extent of residual tumour after neoadjuvant chemoradiotherapy to be an important prognostic factor (13-16). Thus, survival in these patients is closely affected by the response of the primary tumour to such therapy.

It should also be considered that more than half of patients with advanced oesophageal cancers have lymph node (LN) metastasis. The status of this metastasis is an independent and significant prognostic factor in patients who undergo surgical resection without neoadjuvant therapy. In particular, the actual number of involved $\mathrm{LN}$ is one of the most important prognostic factors for patients who undergo surgery alone (17-19). However, the prognostic significance of the number of metastasized LN after neoadjuvant therapy has not been fully elucidated. In addition, it remains unclear how pre-therapy clinical LN status affects survival of patients who undergo chemoradiotherapy followed by surgery. The present study investigated the prognostic significance of both pre- and post-therapy clinical LN status in patients with deeply invading oesophageal squamous cell carcinoma who either underwent chemoradiotherapy followed by surgery or chemoradiotherapy alone.

\section{Materials and methods}

Patients. From April 1994 to December 2006, 195 patients who received chemoradiotherapy as the primary treatment 
for deeply invading thoracic oesophageal carcinoma without distant metastasis [clinical (c T3)-cT4, N0-1, M0] at the Department of Gastroenterological Surgery, Graduate School of Medicine, Osaka University, were included in this study. During the same period, 531 patients with thoracic oesophageal carcinomas underwent surgery with or without preoperative therapy in our institute. All patients had histologically confirmed squamous cell carcinoma of the thoracic oesophagus and received no prior treatment. Patients with distant organ metastasis (stage IVB) were excluded, although patients with cervical LN metastasis were deemed eligible. No patients were over 80 years of age, and all had adequate cardiac, hepatic, renal and bone marrow reserve to tolerate both the planned chemoradiotherapy and the surgical procedures. The study protocol was approved by the Human Ethics Review Committee of Osaka University School of Medicine.

Table I lists the patient characteristics. The median age of patients in this study was 62.3 years (range, 36-80), with 173 men and 22 women. All patients were staged before therapy and postoperatively according to the criteria of the International Union Against Cancer (UICC). Pre-therapy clinical staging was based on oesophagography, endoscopy and computed tomography (CT) of the neck, chest, and upper abdomen using continuous 5-mm-thick slices. Bronchoscopy was performed when tracheobronchial involvement was suspected. From March 2000, positron emission tomography (PET) was also used in our facility for clinical staging where possible. Endoscopic ultrasound (EUS) was not routinely used for staging of locally advanced oesophageal cancers, although it was used in staging superficial oesophageal cancers. Spherical LNs $>1.0 \mathrm{~cm}$ in maximum transverse diameter were diagnosed as metastasis-positive on CT scans $(13,20,21)$. LNs that were visible but $<1.0 \mathrm{~cm}$ on the long axis by CT scan were regarded as metastasis-positive if focal prominent 18-FDG uptake was significant on the PET scan, compared with normal mediastinal activity (22-24).

Treatment regimen. The preoperative chemoradiotherapy regimen in our hospital consisted of simultaneous radiation with administration of 5-fluorouracil (5-FU) and cisplatin as described previously (25-27). 5-FU was administered by continuous intravenous infusion at a dose of $400 \mathrm{mg} / \mathrm{m}^{2}$ in combination with cisplatin at $10 \mathrm{mg} / \mathrm{m}^{2}$ administered by drip infusion for 5 days per week. The planning target volume for radiation therapy was defined as the macroscopic tumour volume plus a $5-\mathrm{cm}$ cephalo-caudal margin and a $2-\mathrm{cm}$ radial margin, including enlarged regional LNs. When the primary tumour was located at the upper third of the thoracic oesophagus, the supraclavicular fossa was included in the radiation field. External-beam radiation therapy was administered by a $10-\mathrm{Mv}$ X-ray linear accelerator with 2 Grays (Gy) per fraction per day and 5 fractions per week for 4-6 weeks, for a total dose of 40-60 Gy. The radiation was generated using parallelopposed fields in an anterior and posterior portal arrangement for 20 fractions followed by oblique or multiple fields for the remaining fractions to spare the spinal cord. In principle, surgery was performed 4-6 weeks after completion of the treatment, when downstaging was achieved by chemoradiotherapy and complete tumour resection was regarded possible.
Table I. Characteristics of 195 patients who received chemoradiotherapy as initial treatment.

$\mathrm{n}(\%)$

\begin{tabular}{lrr}
\hline Age & $62.3(36-80)$ \\
Gender & & \\
Male & 173 & $(89)$ \\
Female & 22 & $(11)$ \\
Tumour location & & \\
Upper third & 74 & $(38)$ \\
Middle third & 93 & $(48)$ \\
Lower third & 28 & $(14)$ \\
Tumour depth & & \\
cT3 & 67 & $(34)$ \\
cT4 & 128 & $(68)$ \\
Lymph node status & & \\
cN0 & 63 & $(22)$ \\
cN1 & 132 & $(68)$ \\
Clinical response & & \\
Complete response & 32 & $(16)$ \\
Partial response & 104 & $(54)$ \\
No change & 57 & $(30)$ \\
Surgery & & \\
Yes & & \\
No & & \\
\hline
\end{tabular}

When tumours were not downstaged or when patients selected chemoradiotherapy alone, surgical resection was not performed. A total of 105 patients received surgical resection: 25 patients underwent transthoracic esophagectomy with two-field lymphadenectomy, 57 underwent transthoracic esophagectomy with three-field lymphadenectomy and 23 patients underwent esophagectomy using the trans-hiatal approach.

Clinical response evaluation. Two weeks after completion of the chemoradiotherapy course, all patients were restaged by endoscopy, CT scan and, in recent cases, PET to evaluate the clinical response to chemoradiotherapy. The response was categorized based on World Health Organization response criteria for measurable disease and the criteria of the Japanese Society for Oesophageal Diseases (28). Complete response (CR) was defined as complete regression of disease. A CR of the primary tumours was determined when tumours disappeared on CT scan and/or PET scan and on endoscopy. If remaining ulceration and/or presence of cancer cells in biopsy samples were confirmed on endoscopy, the case was excluded from the CR category (29). Partial response (PR) was defined by $>50 \%$ reduction in the size of the primary tumour and LN metastasis, as confirmed by CT and endoscopy. Progressive disease (PD) was defined by $>25 \%$ increase 
in the size of the primary tumour or the appearance of new lesions. Cases that did not meet the criteria of PR or PD were defined as no change (NC) $(28,29)$.

Histopathological examination. After fixation in $10 \%$ buffered formalin, the entire surgical specimen was cut into $5-\mathrm{mm}$ slices parallel to the long axis. The tissue slices were embedded in paraffin and cut into at $4-\mu \mathrm{m}$ sections. These thin sections of the primary tumours and LNs were stained with hematoxylin and eosin using routine methods for microscopic examination. Two observers independently examined all specimens. The histopathological findings were classified according to the UICC TNM classification. The degree of histopathological tumour regression in the surgical specimens was classified into five categories. The extent of viable residual carcinoma at the primary site was assessed semiquantitatively, based on the estimated percentage of viable residual carcinoma in relation to the macroscopically identifiable tumour bed that was evaluated histologically. Therapyinduced changes included reactive changes such as necrosis, fibrosis, foamy histiocytes, mucosal oedema, vascular changes in the tumour periphery, and giant cell reactions. Such characteristics were considered signs of neoplastic regression after neoadjuvant chemoradiotherapy $(30,31)$. The percentage of viable residual tumour cells within the total cancerous tissue was assessed as follows: Grade 3, no viable residual tumour cells; Grade 2, <1/3 residual tumour cells; Grade 1b, 1/3-2/3 residual tumour cells; Grade 1a, $>2 / 3$ residual tumour cells; Grade 0, no significant response to chemoradiotherapy $(25,28)$.

Statistical analysis. Overall survival was calculated from the date of preoperative chemoradiotherapy to the occurrence of the event or to the last known date of follow-up. Actual survival was calculated by the Kaplan-Meier method and statistically evaluated by the log-rank test. The Cox proportional hazards regression model was used to analyze the simultaneous influence of prognostic factors. P-values $<0.05$ were considered to indicate statistical significance. These analyses were carried out using the StatView J5.0 software package (Abacus Concepts, Berkeley, CA).

\section{Results}

Clinical response. In 195 patients who received chemoradiotherapy for deeply invading oesophageal squamous cell carcinoma, CR was achieved in 32 cases (16\%) and PR was gained in 104 patients (54\%), while the remaining 57 (30\%) were considered NC. In 163 patients who did not achieve $\mathrm{CR}$, surgical resection after chemoradiotherapy offered survival benefit for those patients (3-year survival rate, 33.1 vs. $0 \%$; Fig. 1). On the other hand, prognosis of the $32 \mathrm{CR}$ patients was not different in the 16 who underwent surgical resection and the remaining 16 who received chemoradiotherapy alone (3-year survival rate, 64.0 vs. $55.7 \%, \mathrm{P}=0.655$; Fig. 1).

Lymph node status before and after therapy. In 105 patients who underwent chemoradiotherapy followed by surgery, overall survival was significantly better in patients who had no LN metastasis before treatment (cNO) than in patients who

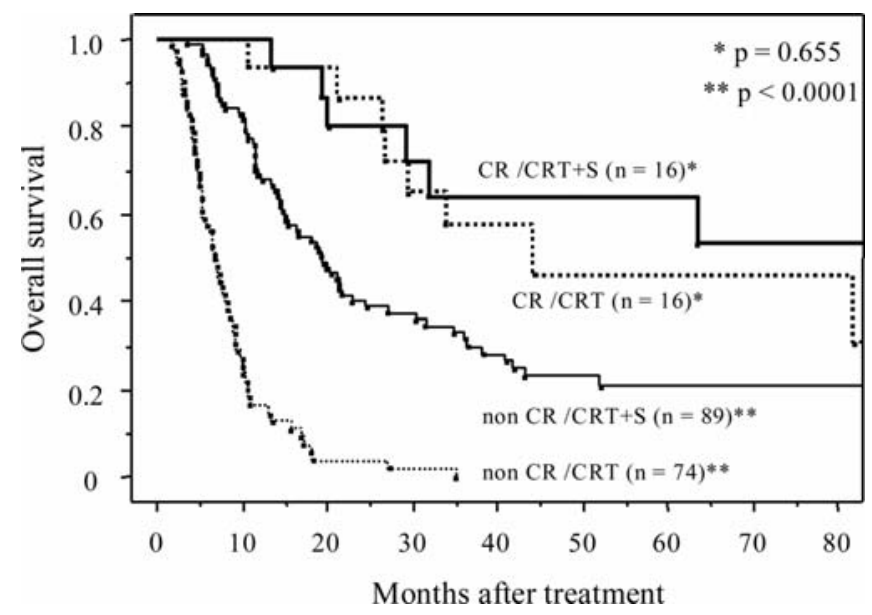

Figure 1. Overall survival of 195 patients, according to clinical response to chemoradiotherapy and surgical resection. CR, complete response; non-CR, partial response, no change and progressive disease. CRT, chemoradiotherapy; $\mathrm{S}$, surgical resection.

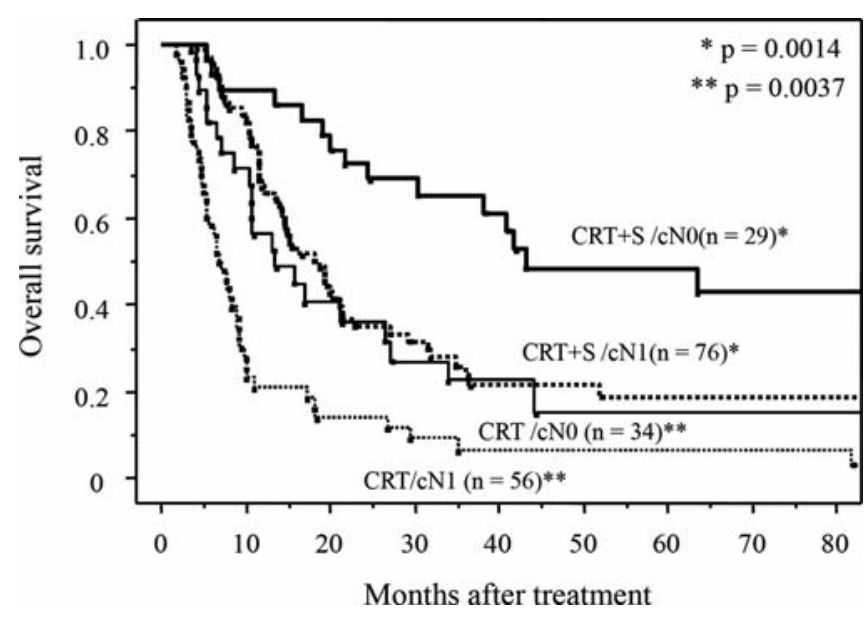

Figure 2. Overall survival of 195 patients according to pre-therapy clinical lymph node status and surgical resection. CRT, chemoradiotherapy; S, surgical resection.

had LN metastasis before treatment (cN1) (3-year survival rate, 65.3 vs. $25.8 \%$; Fig. 2). Similarly, in 90 patients who received chemoradiotherapy alone, overall survival was significantly better in $\mathrm{cN} 0$ patients than in $\mathrm{cN} 1$ patients (3-year survival rate, 22.5 vs. $6.2 \%$; Fig. 2). Thus, pre-therapy LN status significantly affected prognosis of patients, irrespective of receiving surgical resection after chemoradiotherapy.

Next, we investigated the prognostic significance of the number of involved LNs. In 105 patients who underwent surgical resection, the number of involved nodes correlated inversely with overall survival (Fig. 3). The 3-year survival rates were $57.1 \%$ in patients who had no positive LN metastasis, $40.5 \%$ in patients who had 1 positive $\mathrm{LN}$, and $17.6 \%$ in those who had 2-4 positive LNs. However, there was no significant difference in overall survival between patients with $2-4 \mathrm{LN}$ and $>5$ positive nodes.

Prognostic factors affecting survival. Univariate analysis of factors affecting survival of 105 patients who underwent 


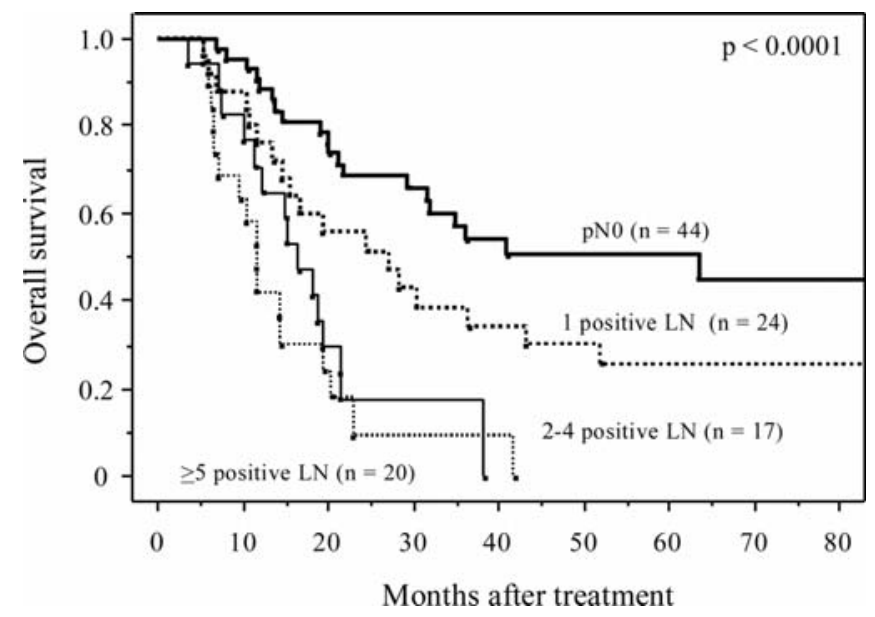

Figure 3. Influence of the number of involved lymph nodes on survival of 105 patients who underwent chemoradiotherapy followed by surgery. LN, lymph node. chemoradiotherapy followed by surgery showed that the number of involved LN, histopathological tumour regression, clinical response, pre-therapy LN status, pathological T stage, and gender were significantly associated with patient prognosis (Table II). Multivariate analysis identified pretherapy LN status and the number of involved LN as the most important independent prognostic factors prior to histopathological tumour regression (Table III). For further analysis, we separated survival curves according to the subclassification of each of these parameters (Fig. 4). Survival was therefore affected by pre-therapy LN status and the number of involved LN, as well as by pre-therapy LN status and histopathological tumour regression.

\section{Discussion}

The number of involved LN correlates closely with poor prognosis in patients who undergo surgery alone. This is

Table II. Univariate analysis of overall survival in 105 patients treated with chemoradiotherapy followed by surgery.

\begin{tabular}{lcccc}
\hline Variables & $\mathrm{n}$ & HR & $95 \% \mathrm{CI}$ & P-value \\
\hline $\begin{array}{l}\text { No. of involved LNs } \\
0-1\end{array}$ & 68 & & & \\
$\quad \geq 2$ & 37 & 3.636 & $2.188-6.024$ & $<0.001$ \\
$\begin{array}{l}\text { Histopathological tumour regression } \\
\quad \text { major R }\end{array}$ & 66 & & & \\
$\quad$ minor R & 39 & 3.108 & $1.876-5.151$ & $<0.001$ \\
Clinical response & & & & \\
$\quad$ CR & 16 & & & \\
NC/PR & 89 & 2.646 & $1.206-5.814$ & 0.015
\end{tabular}

Pre-therapy LN status

cNO 29

cN1 76

$2.506 \quad 1.403-4.484$

0.002

Pathological T stage

pT0-2

pT3-4

Gender

Female

Male

Tumour location

Middle/Lower

Upper

Age

$<70$

$\geq 70$

Clinical T stage

cT3

cT4
$1.524-4.049$

$1.049-6.536$

0.923-.375

$0.719-2.919$

69
0.935

$0.572-1.527$

HR, hazard ratio; CI, confidence interval; CR, complete response; PR, partial response; NC, no change; LN, lymph node; major R, major regression (Grade 2 or 3); minor $\mathrm{R}$, minor regression (Grade 0 or 1). 
Table III. Multivariate analysis of overall survival in 105 patients treated with chemoradiotherapy followed by surgery.

\begin{tabular}{|c|c|c|c|c|}
\hline Variables & $\mathrm{n}$ & HR & $95 \% \mathrm{CI}$ & P-value \\
\hline \multicolumn{5}{|c|}{ No. of involved LNs } \\
\hline $0-1$ & 68 & & & \\
\hline$\geq 2$ & 37 & 2.463 & $1.418-4.274$ & 0.001 \\
\hline \multicolumn{5}{|c|}{ Pre-therapy LN status } \\
\hline cN0 & 29 & & & \\
\hline $\mathrm{cN} 1$ & 76 & 2.299 & $1.264-4.184$ & 0.006 \\
\hline \multicolumn{5}{|c|}{ Histopathological tumour regression } \\
\hline major $\mathrm{R}$ & 66 & & & \\
\hline minor $\mathrm{R}$ & 39 & 1.840 & $1.064-3.184$ & 0.029 \\
\hline \multicolumn{5}{|c|}{ Clinical response } \\
\hline CR & 16 & & & \\
\hline $\mathrm{NC} / \mathrm{PR}$ & 89 & 1.842 & $0.800-4.237$ & 0.151 \\
\hline
\end{tabular}

HR, hazard ratio; CI, confidence interval; CR, complete response; PR, partial response; NC, no change; LN, lymph node; major R, major regression (Grade 2 or 3); minor R, minor regression (Grade 0 or 1 ).
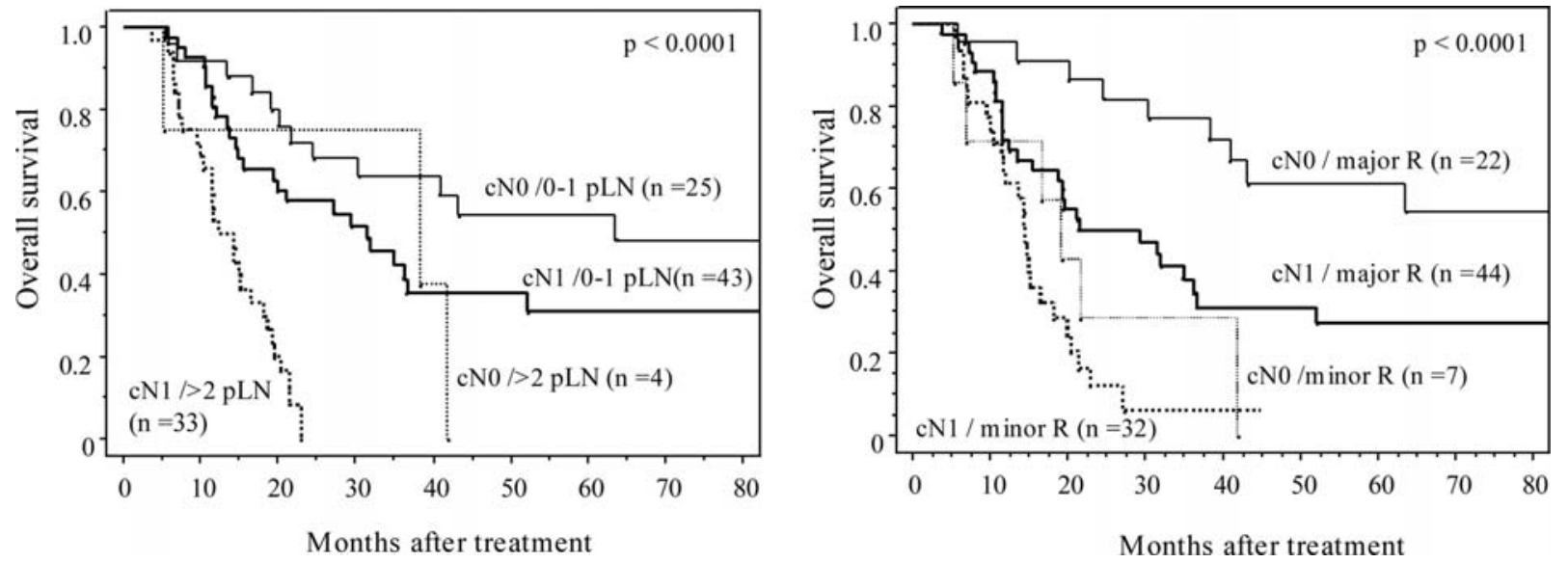

Figure 4. Overall survival of 105 patients who underwent chemoradiotherapy followed by surgery, according to (A) pre-therapy clinical lymph node status and the number of involved lymph nodes or (B) pre-therapy clinical lymph node status and histopathological tumour regression. LN, lymph node; major R, major regression (Grade 2 or 3); minor $\mathrm{R}$, minor regression (Grade 0 or 1 ).

because the number of involved LN influences the incidence of systemic disease in patients with oesophageal cancer (32). However, the prognostic significance of the number of involved LN has not been fully elucidated in the setting of neoadjuvant chemoradiotherapy, with several studies identifying post-therapy pathological nodal status (pN0 or pN1) as a significant independent prognostic factor in such cases $(13,15,33)$. Gu et al reported that overall survival of patients with one metastasis-positive LN was similar to that of patients with no nodal metastasis, but was significantly better than that of patients with $\geq 2$ positive nodes (34). In contrast, Rizk et al demonstrated that the pathological major response of the primary tumour predicts the best survival in squamous cell carcinoma of the oesophagus unlike adenocarcinoma, in which residual nodal disease is the most significant survival predictor
(35). In this study, patients were separated into 4 groups according to involved $\mathrm{LN}(0,1,2-4$ and $\geq 5)$, and survival curves were clearly separated among 0,1 and 2-4 patients. However, survival of patients with $>5$ involved LN was similar to that of patients with 2-4 involved LN. These findings suggested that patients with $\geq 2$ involved LN after neoadjuvant chemoradiotherapy have systemic disease, and may require adjuvant chemotherapy after surgical resection to eradicate systemic micrometastasis.

In the present study, pre-therapy LN status had an equally significant impact on patient prognosis as post-therapy LN status. Chirieac et al reported that post-therapy pathological stage was a better survival predictor than pre-therapy clinical stage (14), while Reynolds et al showed that clinical nodal status did not affect prognosis, but that achieving a node- 
negative status was the major determinant of outcome following neoadjuvant chemoradiotherapy (13). In contrast, Rice et al reported that $\mathrm{cN} 1$ patients who were downstaged to $\mathrm{pN} 0$ by preoperative chemoradiotherapy have a better prognosis than $\mathrm{cN} 1$ patients who were not downstaged, confirming the importance of clinical staging and downstaging (36). The current study revealed a significant difference in prognosis between $\mathrm{cN} 1$ patients and $\mathrm{cN} 0$ patients, despite both showing only 0-1 involved LN after chemoradiotherapy. Moreover, systemic recurrence was more frequent in $\mathrm{cN} 1$ patients compared to cNO patients, albeit with no significant difference in the rate of local recurrence between these patient groups (data not shown). These results indicated that pretherapy LN status might be associated with the extent of systemic disease after chemoradiotherapy irrespective of response to the therapy. From this, it could be recommended that neoadjuvant treatment is modified according to pretherapy LN status to eradicate systemic LN micrometastasis. This might include adding induction chemotherapy prior to the chemoradiotherapy or introducing a more powerful chemotherapy regimen concurrently with radiotherapy.

The present study used CT and FDG-PET, but not endoscopic ultrasound (EUS), to evaluate pre-therapy LN status. EUS has been used widely as a powerful means of assessing clinical $\mathrm{T}$ and $\mathrm{N}$ status, with some studies reporting that EUS is superior to $\mathrm{CT}$ for evaluating regional $\mathrm{LN}$ metastasis and a diagnostic accuracy for LN involvement of approximately $80 \%$ (37-40). However, other studies found that EUS tended to overestimate LN involvement and that the EUS accuracy was operator-dependent due to the experience required to master the technique $(21,41,42)$. In our institute, EUS is routinely performed for staging of superficial oesophageal cancers, but not for deeply invading (T3-T4) oesophageal cancers because the fiberscope often could not pass through the latter tumours due to stenosis. From March 2000, we have used FDG-PET for the clinical staging of locally advanced oesophageal cancers and for evaluating the patient response to neoadjuvant treatment (22-24). FDG-PET reportedly achieves higher specificity and comparable sensitivity for assessing regional and distant $\mathrm{LN}$ involvement, compared with CT and EUS $(21,43,44)$. However, the ability of FDGPET to determine the number of involved $\mathrm{LN}$ remains in question. Nodes less than $8 \mathrm{~mm}$ in diameter are difficult to detect by PET, as is the case with EUS $(21,43,45)$. Future advances in diagnostic techniques are therefore needed to enable more accurate, sensitive, and reliable assessment of involved LN.

There was no survival benefit from surgical resection in the current study for patients who achieved a clinical CR to preoperative chemoradiotherapy, while surgical resection provided significant survival benefit to patients who did not achieve a clinical CR. The retrospective study was clearly a limitation by allowing selection bias, in that indication for surgical resection depends not only on resectability but also on patient selection. Despite this drawback, the current results should be considered in developing a treatment strategy for locally advanced oesophageal cancers. To date, few randomized controlled trials have compared neoadjuvant chemoradiotherapy followed by surgery with chemoradiotherapy alone for advanced oesophageal cancers $(46,47)$. One such study that compared induction chemotherapy followed by chemoradiotherapy with and without surgery found that surgical resection improved local tumour control, although the survival benefit from surgical resection did not reach statistical significance (46). Another study, the EORTC trial (FFCD 9102), compared chemoradiotherapy with surgery to chemoradiotherapy alone for locally advanced resectable oesophageal cancers. It showed no survival benefit from surgical resection for the patients who achieved good response to induction chemoradiotherapy, similar to our result (47). Thus, further studies are required to resolve the question of which patients may truly benefit from surgical resection, and to devise treatments for patients with locally advanced oesophageal cancers based on their response to induction therapy.

In conclusion, the current study demonstrated that pretherapy and post-therapy LN status equally influences survival of patients who receive chemoradiotherapy followed by surgery for deeply invading oesophageal squamous cell carcinoma. In addition, both pre-therapy LN status and the number of post-therapy involved LN may be good indicators of the extent of systemic LN micrometastasis. Thus, control of systemic LN according to pre-therapy and post-therapy LN status may be required, even in patients receiving chemoradiotherapy for deeply invading oesophageal squamous cell carcinoma.

\section{References}

1. Earlam R and Cunha-Melo JR: Oesophageal squamous cell carcinoma: I. A critical review of surgery. Br J Surg 67: 381-390, 1980.

2. Muller JM, Erasmi H, Stelzner M, Zieren U and Pichlmaier H: Surgical therapy of oesophageal carcinoma. Br J Surg 77: 845-857, 1990.

3. Skinner D: Surgical treatment for esophageal carcinoma. Semin Oncol 11: 136-143, 1984.

4. Roder JD, Busch R, Stein HJ, Fink U and Siewert JR: Ratio of invaded to removed lymph nodes as a predictor of survival in squamous cell carcinoma of the oesophagus. Br J Surg 81: 410-413, 1994.

5. Walsh TN, Noonan N, Hollywood D, Kelly A, Keeling N and Hennessy TP: A comparison of multimodal therapy and surgery for esophageal adenocarcinoma. N Engl J Med 335: 462-467, 1996.

6. Burmeister BH, Smithers BM, Gebski V, et al: Surgery alone versus chemoradiotherapy followed by surgery for resectable cancer of the oesophagus: a randomised controlled phase III trial. Lancet Oncol 6: 659-668, 2005.

7. Tepper J, Krasna MJ, Niedzwiecki D, et al: Phase III trial of trimodality therapy with cisplatin, fluorouracil, radiotherapy, and surgery compared with surgery alone for esophageal cancer: CALGB 9781. J Clin Oncol 26: 1086-1092, 2008.

8. Gebski V, Burmeister B, Smithers BM, Foo K, Zalcberg J and Simes J; Australasian Gastro-Intestinal Trials Group: Survival benefits from neoadjuvant chemoradiotherapy or chemotherapy in oesophageal carcinoma: a meta-analysis. Lancet Oncol 8: 226-234, 2007.

9. Urschel JD and Vasan H: A meta-analysis of randomized controlled trials that compared neoadjuvant chemoradiation and surgery to surgery alone for respectable esophageal cancer. Am J Surg 185: 538-543, 2003.

10. Urba SG, Orringer MB, Turrisi A, Iannettoni M, Forastiere A and Strawderman M: Randomized trial of preoperative chemoradiation versus surgery alone in patients with locoregional esophageal carcinoma. J Clin Oncol 19: 305-313, 2001.

11. Berger AC, Farma J, Scott WJ, et al: Complete response to neoadjuvant chemoradiotherapy in esophageal carcinoma is associated with significantly improved survival. J Clin Oncol 23: 4330-4337, 2005. 
12. Rizk NP, Venkatraman E, Bains MS, et al: American Joint Committee on Cancer staging system does not accurately predict survival in patients receiving multimodality therapy for esophageal adenocarcinoma. J Clin Oncol 25: 507-512, 2007.

13. Reynolds JV, Muldoon C, Hollywood D, et al: Long-term outcomes following neoadjuvant chemoradiotherapy for esophageal cancer. Ann Surg 245: 707-716, 2007.

14. Chirieac LR, Swisher SG, Ajani JA, et al: Posttherapy pathologic stage predicts survival in patients with esophageal carcinoma receiving preoperative chemoradiation. Cancer 103 1347-1355, 2005.

15. Schneider PM, Baldus SE, Metzger R, et al: Histomorphologic tumor regression and lymph node metastases determine prognosis following neoadjuvant radiochemotherapy for esophageal cancer. Implication for response classification. Ann Surg 5: 684-692, 2005.

16. Brucher BLDM, Becker K, Lordick F, et al: The clinical impact of histopathologic response assessment by residual tumor cell quantification in esophageal squamous cell carcinomas. Cancer 106: 2119-2127, 2006.

17. Altorki N, Kent M, Ferrara C and Port J: Three-Field lymph node dissection for squamous cell and adenocarcinoma of the esophagus. Ann Surg 236: 177-183, 2002.

18. Akiyama H, Tsurumaru M, Udagawa $\mathrm{H}$ and Kajiyama Y: Radical lymph node dissection for cancer of the thoracic esophagus. Ann Surg 220: 364-373, 1994.

19. Lerut T, DeKeyn P, Coosemsms W, van Raemdonck D, Scheys I and Le Saffre E: Surgical strategies in esophageal carcinoma with emphasis on radial lymphadenectomy. Ann Surg 216: 583-590, 1992.

20. Yano M, Takachi K, Doki Y, et al: Prognosis of patients who develop cervical lymph node recurrence following curative resection for thoracic esophageal cancer. Dis Esophagus 19: 73-77, 2006

21. Lerut T, Flamen P, Ectors N, et al: Histopathologic validation of lymph node staging with FDG-PET scan in cancer of the esophagus and gastroesophageal junction: a prospective study based on primary surgery with extensive lymphadenectomy. Ann Surg 232: 743-752, 2000.

22. Makino T, Doki Y, Miyata H, et al: Use of (18)F-fluorodeoxyglucose-positron emission tomography to evaluate responses to neo-adjuvant chemotherapy for primary tumor and lymph node metastasis in esophageal squamous cell carcinoma. Surgery 144: 793-802, 2008.

23. Higuchi I, Yasuda T, Yano M, et al: Lack of fludeoxyglucose F 18 uptake in posttreatment positron emission tomography as a significant predictor of survival after subsequent surgery in multimodality treatment for patients with locally advanced esophageal squamous cell carcinoma. J Thorac Cardiovasc Surg 136: 205-212, 2008.

24. Miyata H, Doki Y, Yasuda T, et al: Evaluation of clinical significance of $18 \mathrm{~F}$-fluorodeoxyglucose positron emission tomography in superficial squamous cell carcinomas of the thoracic esophagus. Dis Esophagus 21: 144-150, 2008.

25. Yano M, Yasuda T, Miyata H, Fujiwara Y, Takiguchi S and Monden M: Correlation between histological effects on the main tumors and nodal status after chemoradiotherapy for squamous cell carcinoma of the esophagus. J Surg Oncol 89: 244-250, 2005

26. Kishi K, Doki Y, Miyata H, Yano M, Yasuda T and Monden M: Prediction of the response to chemoradiation and prognosis in oesophageal squamous cancer. Br J Surg 89: 597-603, 2002.

27. Miyata H, Doki Y, Shiozaki H, et al: CDC25B and p53 are independently implicated in radiation sensitivity for human esophageal cancers. Clin Cancer Res 6: 4859-4865, 2000.

28. Japanese Society for Esophageal Diseases: Guidelines for the Clinical and Pathologic Studies on Carcinoma of the Esophagus. 10th edition. Kanehara Syuppan, Tokyo, 2007.

29. Ohtsu A, Boku N, Muro K, et al: Definitive chemoradiotherapy for T4 and/or M1 lymph node squamous cell carcinoma of the esophagus. J Clin Oncol 17: 2915-2921, 1999.
30. Mandard AM, Dalibard F, Mandard JC, et al: Pathologic assessment of tumor regression after preoperative chemoradiotherapy of esophageal carcinoma. Cancer 73: 2680-2686, 1994.

31. Chang F, Deere H, Mahadeva U and George S: Histopathologic examination and reporting of esophageal carcinomas following preoperative neoadjuvant therapy; Practical guidelines and current issues. Am J Clin Path 129: 252-262, 2008.

32. Peyre CG, Hagen JA, DeMeester SR, et al: Predicting systemic disease in patients with esophageal cancer after esophagectomy: a multinational study on the significance of the number of involved lymph nodes. Ann Surg 248: 979-985, 2008.

33. Gaca JG, Petersen RP, Peterson BL, et al: Pathologic nodal status predicts disease-free survival after neoadjuvant chemoradiation for gastroesophageal junction carcinoma. Ann Surg Oncol 13: 340-346, 2006.

34. Gu Y, Swisher SG, Ajani JA, et al: The number of lymph nodes with metastasis predicts survival in patients with esophageal or esphagogastric junction adenocarcinoma who receive preoperative chemoradiation. Cancer 106: 1017-1025, 2006.

35. Rizk NP, Seshan VE, Bains MS, Ilson DH, Minsky BD, Tang L and Rusch VW: Prognostic factors after combined modality treatment of squamous cell carcinoma of the esophagus. J Thorac Oncol 2: 1117-1123, 2007.

36. Rice TW, Blackstone EH, Adelstein DJ, et al: N1 esophageal carcinoma: the importance of staging and downstaging. J Thorac Oncol 121: 454-464, 2001

37. Kelly S, Harris KM, Berry E, et al: A systematic review of the staging performance of endoscopic ultrasound in gastrooesophageal carcinoma. Gut 49: 534-539, 2001.

38. Botet JF, Lightdale CJ, Zauber AG, Gerdes H, Urmacher C and Brennan MF: Preoperative staging of esophageal cancer: comparison of endoscopic US and dynamic CT. Radiology 181: 419-425, 1991.

39. Tio TL, Coene PP, Luiken GJ and Tytgat GN: Endosonography in the clinical staging of esophagogastric carcinoma. Gastrointest Endosc 36: S2-S10, 1990.

40. Greenberg J, Durkin M, van Drunen M and Aranha GV: Computed tomography or endoscopic ultrasonography in preoperative staging of gastric and esophageal tumors. Surgery 116: 696-701, 1994.

41. Vickers $J$ and Alderson D: Oesophageal cancer staging using endoscopic ultrasonography. Br J Surg 85: 994-998, 1998.

42. Van Vliet EP, Eijkemans MJ, Poley JW, Steyerberg EW, Kuipers EJ and Siersema PD: Staging of esophageal carcinoma in a low-volume EUS center compared with reported results from high-volume centers. Gastrointest Endosc 63: 938-947, 2006.

43. Kato H, Kuwano H, Nakajima M, et al: Comparison between positron emission tomography and computed tomography in the use of the assessment of esophageal carcinoma. Cancer 94: 921-928, 2002

44. Flamen P, Lerut A, van Cutsem E, et al: Utility of positron emission tomography for the staging of patients with potentially operable esophageal carcinoma. J Clin Oncol 18: 3202-3210, 2000.

45. Luketich JD, Schauer P, Landreneau R, et al: Minimally invasive surgical staging is superior to endoscopic ultrasound in detecting lymph node metastases in esophageal cancer. J Thorac Cardiovasc Surg 114: 817-821, 1997.

46. Stahl M, Stuschke M, Lehmann N, et al: Chemoradiation with and without surgery in patients with locally advanced squamous cell carcinoma of the esophagus. J Clin Oncol 23: 2310-2317, 2005.

47. Bedenne L, Michel P, Bouche O, et al: Chemoradiation followed by surgery compared with chemoradiation alone in squamous cancer of the esophagus: FFCD 9102. J Clin Oncol 25: 1160-1168, 2007. 